\title{
Transferencia Plasmidial de Multirresistencia en Cepas Enteropatógenas de Escherichia Coli Serogrupo 0111
}

\author{
T.M. M. Eliana Bustos M.; T.M. Miriam Troncoso H.; M.A. M. Soledad Toledo B.; \\ T.M. Guillermo Figueroa $\mathrm{G}^{1}$ \\ Transference of multiresistance to antibiotics by \\ plasmids in enteropathogenic $0111 \mathrm{E}$. Coli.
}

\begin{abstract}
Susceptibility to antıbiotics was studied in 38 strains of enteropathogenic E. coli 0111 obtained from chitdren under 2 years of age, 29 of these were obtained from patients with aeute diarrhoea and the remainder from asymptomatic children. Fifty tive percent of the strains were resistant to three or more antibiotics: $100 \%$ of these were resistant to ampicillin, $90 \%$ to kanamycin and carbenicillin, approxinately $80 \%$ to streptomycin and cephalothin but they were ald sensitjve to furazolidone and $90 \%$ to gentamicin. In vitro conjugation tests showed that $34 \%$ of the multiresistant strains were capable of transfering 2 to 6 resistance traits. Molecular weights of plasmids assaciated to transference ranged between 41 and 80 Megadaltons (Md), but among the strains isolated from patients with acute diarthoea there was a clear predominance of 54 to $55 \mathrm{Md}$ plasmids. No differences were demonstrated in the frecuency of multiresistance and/or the transference capacity of strains isolated from patients with dianhoea and those coming from asymptomatic children.

(Key words: Antibiotic tesistance. Enteropathogenic E. Coli. Plasmidial transference).
\end{abstract}

La Escherichia coli enteropatógena serogrupo 0111 (ECE-0111) ha sido asociada a brotes epidémicos y cuadros de enteritis infantil desde la década del $40^{1-2}$ hasta hoy; sin embargo, el o los mecanismos involucrados en dicha capacidad enteropatógena no han sido totalmente dilucida. dos.

Diversos estudios han demostrado que ECE-011 I no invade la mucosa intestinal. ni produce las enterotoxinas termoestable (ST) y termolábil $(\mathrm{LT})^{3-5}$. En este grupo se ha detectado sin embargo un factor citotóxjco capaz de estimular la adenil-ciclasa in vitro que tiene efecto biológico en el asa jejunal de conejo ${ }^{6}$. La actividad citotóxica ha sido estudiada en células Vero ${ }^{7}$, demostrándose que sôlo cierta proporción de las cepas enteropatógenas de ECE-O111 ensayadas producen efecto sobre ellas, lo que sugiere que la citotoxicidad no seria común a todas las cepas.

Otros investigadores han demostrado una peculiar capacidad de las cepas de este serogrupo

1. Instituto de Nutrición y Tecnología de los Alimentos, Universidad de Chile, Santiago.

Trabajo financiado parcialmente por el Proyecto No 1764-852 F del Departamento de Investigación y Bibliotecas de la Universidad de Chile. para adherirse a la mucosa intestinal, originando en ella cambios morfológicos y físiológicos ${ }^{8}$. La capacidad adhesiva ha sido confirmada in vitro mediante ensayos en tineas celulares $\mathrm{HEp}-2^{9} y$ Hela10. Al parecer la adherencia específica estaría ligada a la presencia en la bacteria de un plasmidio de pe so molecular entre 50 y $70 \mathrm{Md}^{11}$.

En Chile, la ECE-0111 es, entre los denominados "serotipos clásicos", uno de los más frecuentemente aislados de ninos con diarrea aguda epidémica 0 esporádica ${ }^{12-13}$. Estudios preliminares en nuestro medio han demostrado que este serogrupo presenta una elevada resistencia a los antibióticos ${ }^{14}$, generalmente asociada a plasmidios, pues ha sido posible transferirla mediante ensayos de conjunción in vitro.

Este estúdio se hizo con el propósito de cstudiar la frecuencia de cepas multirresistentes de E. coli serogrupo 0111; la capacidad de transferencia conjugacional de uno o más de los determinantes asociados con la resistencia; y si existen diferencias en dichos parámetros entre cepas provenientes de casos con diarrea o de niños aparentemente sanos (portadores).

\section{MATERIAL Y METODOS}

Entre Enero 1981 y Dicjembre 1984 se estu- 
diaron 38 cepas de E. coli enteropatógenas serogrupo 0111 aisladas de coprocultivos de niños menores de dos años, 29 provenían de pacientes con diarrea y 19 niños asintomáticos. En e] momento de tomar las muestras ningún niño estaba hospitalizado ni había recibido antibióticos en las últimas 2 semanas.

Las cepas de E. coli fueron tipificadas con sueros poli y monovalentes 0 (Difco). La susceptibilidad a los antibióticos fue eștudiada con el mètodo de difusión en agar de Bauer ${ }^{15}$ utilizando sensidiscos comerciales ( $\mathrm{BBL}$ ).

Los ensayos de transferencia genética a traves de conjugación in vitro, se realizaron según la técnica de Miller ${ }^{16}$ usando cono cepa receptora E. coli $\mathrm{K} 12$ resistente a ácido nalidíxico (EC K12-RAN).

EI las cepas multirresistentes y sus transconjugantes se determinó la concentración inhibitoria minima (CIM) según los métodos estandar de dilución en agar de Ericson y Sherris ${ }^{17}$.

Todas las cepas multirresistentes de ECE-0111. asi como sus correspondientes transconjugantes, fueron sometidas a estudios de Acido Desoxirribo Nuclejco (ADN) plasmidial según el método propuesto por Kado $y \operatorname{Lin}^{18}$; las bacterias fueron destruídas con una solución $\mathrm{dc} 50 \mathrm{mM}$ (Hidro. ximetil) aminometano (Tris) y 3\% Docecil sulfato de Sodio (SDS) (pH 12.6) y la extracción del ADN se hizo con una mezcla de fenol-cloroformo $(1: 1 . v: v)$. Las corridas electroforéticas se llevaron a cabo durante 4 horas a 50 volts, con el gel sumergido en Tampón Tris-Borato $(\mathrm{pH}$ 7.719. Los geles se tin̄eron con Bromuro de Etidio y se observaron sobre una cámara con luz ultravioleta, fotografiándose con pelicula Pola. roid tipo 55.

El peso molecular (PM) de los plasmidios se calculó según el método de Meyers ${ }^{20}$ que consiste en comparar la distancia recorrida por ellos con la de plasmidios de PM conocido durante la corrida electroforética. Los plasmidios utilizados y sus PM expresados en Megadaltons (Md) fueron: Plac (101 Md), Rl (61 Md), RP 4 (36 Md) y $\mathrm{SA}(26 \mathrm{Md})$.

\section{RESULTADOS}

De las 38 cepas de ECE-0111 estudiadas, 2I $(55,3 \%)$ presentaron resistencia simultánea a 3 ó más antibióticos, $17(44,7 \%)$ habían sido aisladas de casos de diarrea y $4(10,5 \%)$ de niños asintomáticos; las diferencias no eran estadísticamente significativas ( $\rho>0,20$ prueba exacta de Fisher).

El comportamiento de las cepas multirresistentes frente a 12 antimicrobianos se muestra en

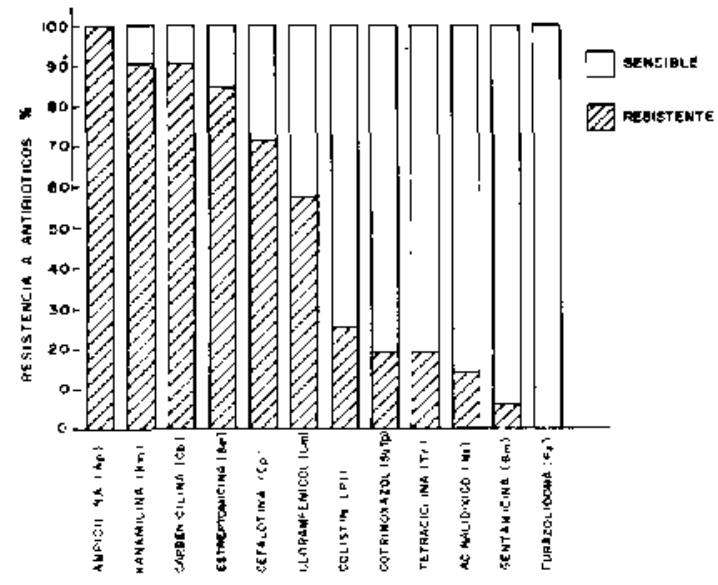

Figura 1: Susceptibilidad de 21 cepas de E. coli 0111 multirresistencia a 12 antibioticos

la Figura 1. Los niveles de resistencia de las cepas parentales mostraron CIM iguales o mayores a $128 \mu \mathrm{g} / \mathrm{ml}$ para ampicilina, cloramfenicol, estreptomicina y cotrimoxazol.

Las cepas de E. coli 0111 que mostraron resistencia múltiple albergaban de 1 a 4 plasmidios, cuyos pesos moleculares calculados estaban en un rango de 28 a $107 \mathrm{Md}$ (Tabla 1 ).

Trece (34\%) cepas multirresistentes de E. coli oll1 transfirieron algún determinante de resistencia mediante collugación, 10 provenían de pacientes con diarrea y 3 de niños asintomáticos $(\mathrm{p}=\mathrm{NS})$; la frecuencia de transferencia de los marcadores genétjcos estudiados fluctuó para las distintas cepas entre $7.29 \times 10^{-5}$ y $6.25 \times 10^{-9}$.

Los patrones de resistencia, el número de plasmidios identificados en cada cepa y los pesos moleculares calculados de éstos, tanto para las cepas parenterales como para sus transconjugan. tes, se muestran en la Tabla 2 .

La resistencia de las cepas transconjugantes, comparadas con las de las parentales fue seme. jante para la ampicilina en $60 \%$ y menor en $40 \%$; La CIM del cloramfenicol se mantuvo en la mayoría de las cepas $(87,5 \%)$, aumentando en una $(12,5 \%)$; la de cotrimoxazol se mantuyo en todos los casos (100\%), en cambio la CIM para estreptomicina sólo se mantuvo en $40 \%$ y disminuyó en el 60\% restante.

Las cepas multirresistentes transfirieron en bloque de 2 a 6 determinantes genéticos de resistencia.

Los estudios de coelectroforésis del ADN plas. midial demostraron que las cepas de F95, 015, $03, A 11,42,947,34$ y 102, que portaban un plasmidio de $54-55 \mathrm{Md}$, comigraban originando una sola banda en los geles. 


\section{Tabla 1}

Patrones de resistencia, número de plasmidios identificados y sus PM en cepas multirresistentes de Escherichia coli 0111

\begin{tabular}{|c|c|c|c|}
\hline $\begin{array}{l}\text { No } \\
\text { Cepa }\end{array}$ & Pa1rón de Resistencia* & $\begin{array}{l}\text { No Plasmidios } \\
\text { Identificados }\end{array}$ & PM (Md) Plasmidios \\
\hline A274 & $A p-S m-C b$ & 1 & 42 \\
\hline $\mathrm{C} 225$ & $\mathrm{Ap}-\mathrm{Sm}-\mathrm{Cb}-\mathrm{Tc}-\mathrm{Km}$ & 1 & 47 \\
\hline 791 & $\mathrm{Ap}-\mathrm{Sm}-\mathrm{Cb}-\mathrm{Cp}-\mathrm{Km}-\mathrm{Pl}-\mathrm{Cm}$ & 1 & 107 \\
\hline AS2 & $\mathrm{Ap}-\mathrm{Km}-\mathrm{Nx}$ & 2 & $29-42$ \\
\hline $\mathrm{Cl2}$ & $\mathrm{Ap}-\mathrm{Sm}-\mathrm{Cp}-\mathrm{Tc}$ & 2 & $32-87$ \\
\hline 20 & $\mathrm{Ap}-\mathrm{Sm}-\mathrm{Cb} \cdot \mathrm{Cm}-\mathrm{Km}$ & 2 & $45-61$ \\
\hline 18 & $\mathrm{Ap}-\mathrm{Cb}-\mathrm{K} \mathbf{m}-\mathrm{Cp}$ & 2 & $36-48$ \\
\hline $\mathrm{C} 32$ & $\mathrm{Ap}-\mathrm{Sm}-\mathrm{Cb}-\mathrm{Cp}-\mathrm{Km}-\mathrm{Pl}$ & 2 & $32-47$ \\
\hline H11 & $\mathrm{Ap}-\mathrm{Sm}-\mathrm{Cb}-\mathrm{SuTp}-\mathrm{Km}-\mathrm{Pt}$ & 2 & $28-41$ \\
\hline 195 & $\mathrm{Ap}-\mathrm{Cm}-\mathrm{Cb}-\mathrm{Km}$ & 3 & $32-42-55$ \\
\hline 34 & $\mathrm{Ap}-\mathrm{Cm}-\mathrm{Cb}-\mathrm{Km} \sim \mathrm{Cp}$ & 3 & $30-42-55$ \\
\hline 015 & $A p-C m-C b-K m-S m-C p$ & 3 & $32 \cdot-42-55$ \\
\hline 03 & $\mathrm{Ap}-\mathrm{Cm}-\mathrm{Cb}-\mathrm{Km}-\mathrm{Sm}-\mathrm{Cp}$ & 3 & $32-42-55$ \\
\hline$A 11$ & $\mathrm{Ap}-\mathrm{Cm}-\mathrm{Cb}-\mathrm{Km}-\mathrm{Sm}-\mathrm{Cp}$ & 3 & $30-41-54$ \\
\hline 42 & $\mathrm{Ap}-\mathrm{Cm}-\mathrm{Cb}-\mathrm{Km}-\mathrm{Sm} \cdot \mathrm{Cp}$ & 3 & $30-41-54$ \\
\hline 16 & $A p-C b-K m-S m-S u T p \cdot P I$ & 3 & $29-40-66$ \\
\hline 947 & $A p-C m-C b-K m-S m-C p-N x$ & 3 & $30-41-54$ \\
\hline 27 & $\mathrm{Ap}-\mathrm{Cn}-\mathrm{Cb}-\mathrm{Km}-\mathrm{Sm}-\mathrm{Cp}-\mathrm{Tc}$ & 3 & $28-39-80$ \\
\hline CZ10 & $\mathrm{Ap}-\mathrm{Cm}-\mathrm{Cb}-\mathrm{Km}-\mathrm{SuTp}-\mathrm{Cp}-\mathrm{Nx}$ & 3 & $32-47-61$ \\
\hline 102 & $\mathrm{Ap}-\mathrm{Cm}-\mathrm{Cb}-\mathrm{Km}-\mathrm{Cp} \cdot \mathrm{Gm}$ & 4 & $32-36-43 \cdot 55$ \\
\hline 79 & $\mathrm{Ap}-\mathrm{Cb}-\mathrm{Km} \cdot \mathrm{Sm}-\mathrm{SuTp} \mathrm{Tc}$ & 4 & $30-36-40-45$ \\
\hline
\end{tabular}

* Los símbolos de los antibióticos corresponden a los empleados en la Fìgura 1.

Tabk 2.

Patrones de tesisfencia, número de plasmidios identifieados y determinantes transferidos por cepas enteropatógenas de É. coli 011 l multirresistentes a los antibióticos

\begin{tabular}{|c|c|c|c|c|c|}
\hline $\begin{array}{l}\text { No de } \\
\text { Cepa }\end{array}$ & $\begin{array}{l}\text { Patrón de Resistencia * } \\
\text { Cepas Parentales }\end{array}$ & $\begin{array}{l}\text { Na Plasmidios } \\
\text { Identíficados }\end{array}$ & $\begin{array}{l}\text { PM. (Mfd) } \\
\text { Plasmidios }\end{array}$ & $\begin{array}{l}\text { Patrón de Resistencia } \\
\text { Cepas Transferidas }\end{array}$ & $\begin{array}{l}\text { P.M. (Md) } \\
\text { Plasmidios } \\
\text { Transferidos }\end{array}$ \\
\hline F95 & $\mathrm{Ap} \mathrm{Cm} \mathrm{Cb} \mathrm{Km}$ & 3 & $55-42-32$ & $\mathrm{Cm} \mathrm{km}$ & 55 \\
\hline 015 & Ap Cm Cb Km Sm Cp & 3 & $55 \cdot 42-32$ & $\mathrm{Cm} \mathrm{Km}$ & 35 \\
\hline 013 & $\mathrm{Ap} \mathrm{Cn} C b \mathrm{Km} \mathrm{Sm} \mathrm{Cp}$ & 3 & $55 \quad 42-32$ & $\mathrm{Cm} \mathrm{Km}$ & $\$ 5$ \\
\hline 18 & $\mathrm{Ap} \mathrm{Cb} \mathrm{Km} \mathrm{Cp}$ & 2 & $48-36$ & $\mathrm{Ap} \mathrm{CbCp}$ & 48 \\
\hline 16 & $\mathrm{Ap} \mathrm{Cb} \mathrm{Km} \mathrm{Sm} \mathrm{SuTp} \mathrm{Pl}$ & 3 & $66-40-29$ & $\mathrm{AP} \mathrm{Cb} \mathrm{Sm}$ & 66 \\
\hline H1 1 & $A p C b K m$ Sm SuTp Pl Tc & 2 & $41 \quad 28$ & $\mathrm{Ap} \mathrm{Cb} \mathrm{Sm}$ & 41 \\
\hline 79 & Ap Cb Km Sm SuTp PI Tc & 4 & $4540 \cdot 36-30$ & $\mathrm{Ap} \mathrm{Cb} \mathrm{Sm} \mathrm{Cp}$ & 45 \\
\hline A]1 & Ap $C m C b \mathrm{Km} \mathrm{Sm} \mathrm{Cp}$ & 3 & $5441-30$ & $\mathrm{Cm} \mathrm{Km} \mathrm{Sm}$ & 54 \\
\hline 42 & Ap $\mathrm{Cm} \mathrm{Cb} \mathrm{Km} \mathrm{Sm} \mathrm{Cp}$ & 3 & $54-4130$ & $\mathrm{Cm} \mathrm{Km} \mathrm{Sm}$ & 54 \\
\hline 947 & Ap $\mathrm{Cm} C b \mathrm{Km} S \mathrm{Sm} \mathrm{Nx}$ & 3 & $54-41-30$ & Cm Km Sn & 54 \\
\hline 34 & $\mathrm{Ap} \mathrm{Cm} \mathrm{Cb} \mathrm{Km} \mathrm{Cp}$ & 3 & $5542 \cdot 30$ & $\mathrm{Cm} \mathrm{Km} \mathrm{Cb}$ & 55 \\
\hline 102 & Ap $\mathrm{Cm} \mathrm{Cb} \mathrm{Km} \mathrm{Cp} \mathrm{Gm}$ & 4 & $55-43-36 \quad 32$ & $\mathrm{Ap} \mathrm{Cm} \mathrm{Km} \mathrm{Cb} \mathrm{Cp} \mathrm{Gm}$ & $\$ 5$ \\
\hline 27 & $\mathrm{Ap} \mathrm{Cm} \mathrm{Cb} \mathrm{Km} \mathrm{Cp} \mathrm{Sm} \mathrm{Tc}$ & 3 & $8039-28$ & $\mathrm{Ap} \mathrm{Cm} \mathrm{Km} \mathrm{Cb} \mathrm{Sm} \mathrm{Tc}$ & 80 \\
\hline
\end{tabular}

* Los símbolos de los antibiótícos corresponden a los empleados en la Figura 1.

\section{DISCUSION}

Los resultados del estudio de 38 cepas de $\mathbf{E}$. coli enteropatógeno serogrupo 0111 muestran que hay un elevado numero de cepas multirresistentes $(21 / 38)$, en su mayoria (13/21) capaces de transferir por lo menos un plasmidio asociado a la resistencia. La multirresistencia se detectó con igual frecuencia en las cepas provenientes de niños con diarrea y asintomáticos, lo que sugiere una gran diseminación de cepas multirresistentes en nuestro medio. Este hecho tiene gran impor. 
Tabla 3

Antibióticos que se transfirieron en bloque mediante conjugación en cepas multirresistentes de E. coli enteropatógenas 0111 .

\begin{tabular}{cll}
\hline $\begin{array}{l}N^{\circ} \mathrm{dc} \\
\text { antibioticas }\end{array}$ & $\begin{array}{l}\mathrm{N}^{0} \mathrm{de} \\
\text { cepas (13) }\end{array}$ & $\begin{array}{l}\text { Bloque de } \\
\text { resistencia }\end{array}$ \\
\hline 2 & $3(23 \%)$ & $\mathrm{Cm} \mathrm{Km}$ \\
3 & 3 & $\mathrm{Cm} \mathrm{Km} \mathrm{Sm}$ \\
4 & 3 & Ap Cb Sm SuTp \\
6 & $2(15 \%)$ & Ap Cb Km Cm Cp Gm \\
\hline
\end{tabular}

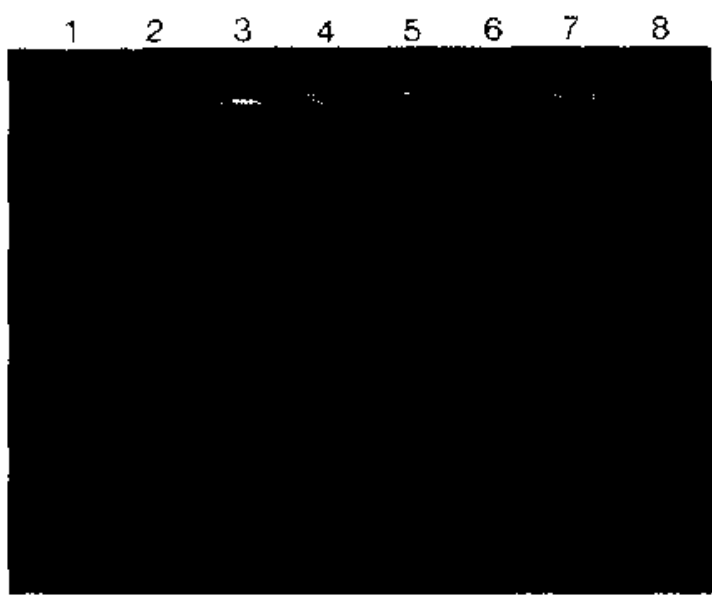

Figura 2. Electroforesis en get de agarosa del DNA plasmidial de diferentes cepas de E. coli 0111 parentales y su correspondiente transconjugante. Linea 1: 03, línea 2: transconjugante. Linea 3; 015, línea 4: transconjugante.

Linea 5: Plac (10I Md) y RP4 (36 Md).

Linea 6: RI (61 Md) y SA (26 Md).

Linea 7: F95, línea 8: transconjugante.

tancia, ya que se sabe que la presencia de plasmidios $\mathbf{R}$ en las bacterias puede aumentar su viru. lencia ${ }^{2}$. Por otra parte, desde el punto de vista ecológico es posible el traspaso de estos determinantes de resistencia a cepas avirulentas de la flora intestinal. situación en que, eventualmente, pudieran transformarse en agentes potencialmente patógenos fuera del tubo digestivo, $o$ en reservorios del material genético de la cepa original 22

Además, se podría especular que la presencia de plasmidios $R$ podría constituirse en un mecanismo útil para movilizar otros plasmidios que codifican para diferentes propiedades bacterianas 23

El patrón de resistencia plasmidial de las cepas parentales era muy parecido en el número y peso molecular de los plasmidios, en 8 de 13 cepas enteropatógenas multirresistentes, hecho que se verificó al efectuar coeletroforésis con las transconjugantes, ya que los plasmidios de éstas comigraban originando una sola banda. El fenómeno es interesante, pues las cepas fueron aisladas de coprocultivos tomados de distintos individuos, a lo largo de 3 años, con varios meses de intervalo y provenientes de diferentes sitios geográficos de la ciudad, lo que sugiere que la mayoria de estas cepas tienen un origen clonal común.

Llama la atención la repetición de plasmidio de 54-55 $\mathrm{Md}$ en cepas provenientes de casos de diarrea (6/8). Este plasmidio podría corresponder a un elemento genético que codifique la capacidad de adherencia a las células intestinales $8-11$ $y$ permita como ha sido demostrado, que estas cepas se adhieran "in vitro" a células de lineas Hela y HEp-2. Estudios en este sentido serán motivo de una comunicación posterior. Es interesante anotar que plasmidios de $54-55 \mathrm{Md}$ no se detectaron en las cepas multirresistentes que no transfirieron su material génetico, lo que respaldaría la posibilidad de que dicho elemento fuese un plasmidio conjugativo.

Nuestros resultados indican que, generalmente, la multirresistencia se transficre en bloque.

\section{RESUMEN}

Se estudió la sensibilidad de los antibióticos de 38 cepas enteropatógenas de Escherichia coli 01 l l provenientes de niños menores de dos años, 29 obtenidas de pacientes con diarrea, las restantes de sujetos asintomáticos. Se encontró que $55 \%$ de las cepas eran resistentes a 3 o más antimicrobianos simultáneamente; de ellas todas lo eran a ampicilina, $90 \%$ a kananicina y carbenicilina $y$ alrededor de $80 \%$ a estreptomicina $y$ cefalotina; todas las cepas eran sensibles a fura. zolidona y $90 \%$ a gentamicina.

Entre las cepas multirresistentes, $34 \%$ eran capaces de transferir, mediante conjugación, 2 a 6 deternunantes de resistencia. EI peso molecular de los plasmidios asociados a la transferencia flutuaba entre 41 y 80 Megadaltons (Md), predominando el de 54-55 Md. No se encontraron diferencias significativas en la frecuencia de multirresistencia y la capacidad de transferir entre cepas provenientes de nintos con diarrea y asintomáticos.

\section{REFERENCIAS}

1. Bray, J.: Isolation of antigenically homogeneous strains of Bact. coli neopolitanum from summer diarrhoea of infants. J. Pathol. 57: 239, 1945. 
2. Neter, E., Korns, R.F., Trussell, R.E.: Association of Escherichia coli 0111 with two hospital outbreaks of epidemic diarthoea of the newborn infant in New Yotk State during 1947. Pediatr. 12: 377,1953

3. Echeverria, P., Chang, $C$. Smith, D., Anderson, G.: Enterotoxigenety and invasive capacity of "enteropathogenic" serotypes of Escherichia coli.J. Pediatr. 89: $8,1976$.

4. Gurwith, M. Wiseman, D., Chow. P.: Clinical and Laboratory Assessment of the Pathogenlcity of Serotyped Enteropathogenic Escherichia coli. J. Infect. Dis. 135: 736, 1977.

5. Goldschmidt. M., Du Pont, H.: Enteropathogenic Escherichia coli: lack of correlation of serotype with pathogenicity. J. Infect. Dis. 133: 153, 1976.

6. Schefiel, J., Martin, C., Bober, C., Monteil, H.: Isolation of an enterotoxic factor elaborated by human enteropathogenic Escherichia coli. FEMS Microbiol. Lett. 9: 125, 1980.

7. Karmali, M., Steele, B., Petric, M., Lim. C.: Sporadic cases of haemolytic-uremic syndrome associated with faecal cytotoxin and cytotoxinproducing Escherichia coli in stools. Lancet, 19: $619,1983$.

8. Clousen, C. Christie, D.: Chtonic diarthoea in infants caused by adherent enteropathogenic Escherichía coli. J. Pediatr. 100: 358, 1982.

9. Cravioto, A., Gross, R.J., Scotiand, S., Rowe, B.: An adhesive factor found in strains of Escherichia coli belonging to the traditional infantile enteropathogenic serotypes. Curr. Microbiol., 3: 95, 1979.

10. Scaletsky. I., Silva, M.L., Trabulsi, L.: Distintive patterns of adherence of enteropathogenic Escherichia coli to Hela cells. Infect Immon. 45: $534,1984$.

11. Baldini, M., Kaper, J., Levine, M., Candy, D., Moon, H.: Plasmid Mediated adhesion in enteropathogenic Escherichia coli. J. Pediatr. Gastroenterol. Tuts. 2: 534,1983

12. Figueroa, G: Estudio de prevalencia de serotipos enteropatógenos de Eschirichia coli en deposiciones de nifios que habitan una población suburbana de Santiago. Rev, Chil. Pediatr. 51: 225, 1980.

13. Prado, V., Braten, S, Bosch, P., Bercovich, $M$. Reyes, L., Sawado, M.: Análisis de Escherichia colí enteropatógeno clásico (E.C.E.P.) como causa endémica đe diartea aguda en niños chilenos. Rev. Chil. Pediatr. 55: 171, 1984.

14. Figteroa, G., Troncoso, M., Araya, M. Espinoza, J., Brunser, O.: Enteropathogen carriage by healthy individuals living in an area with poor sanitation. $J$. Hyg. (Camb.), 91: 499, 1983.

15. Bauer, A.+ Kirby, W., Sherris, J., Turk, M.: Antibjotic susceptibility testing by a standarized single disk me thod. Am. J. Clin. Pathol. 45: 493, 1966.

16. Curtis, $R$. III Gene Transfer Manual of Methods for General Bacteriology (ed. P. Gerhardt). pp. 243. American Society for Microbiology (ed) Washington, D.C., 1981 .

17. Ericsson, H., Sherris, $f_{-:}$Antibiotic sensitivity testing. Report of an Internationai Collaborative Study. Acta Pathol. Microbiol. Scand. B. Suppl. 217: 1, 1971 .

18. Kodo, C. Liu, S. Rapid procedure for detection and isolation of large and small plasmids. $J$. Bateriol. 145: 1365, 1981

19. Maniatis, T., Fritsch., Sambrook, $t$.: Molecular cloning a laboratory manual. coli Spring Harbor Laboratory (ed) N. York. p.156, 1982.

20. Meyers, I.A., Sanchez, D., Elwell, LP., Falkow, S.: Simple agarose gel electrophoretic method for the idertification and characterization of plasmid deoxyribonucleic acid. J. Bacteriol. 127: 1529 . 1976.

21. Elwell, L.: Plasmid-mediated factors associated with virulence of bacteria to animals. Ann. Rev. Microbiol. 34: 465, 1980.

22. Burt, $S$., Woods, $D$.: R. Factor transfer to obligate anaerobes from Escherichia coli. J. Gen. Microbjol., 93: $405,1976$.

23. Anderson, E.S.: Plasmid transfer in Escherichia coli. J, Infect. Dis., 137: 686, 1978. 